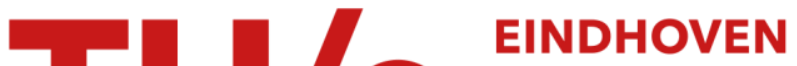 UNIVERSITY OF TECHNOLOGY
}

\section{Sensing Methodology for the Rapid Monitoring of Biomolecules at Low Concentrations over Long Time Spans}

\section{Citation for published version (APA):}

Lubken, R. M., Bergkamp, M. H., de Jong, A. M., \& Prins, M. W. J. (2021). Sensing Methodology for the Rapid Monitoring of Biomolecules at Low Concentrations over Long Time Spans. ACS Sensors, 6(12), 4471-4481. https://doi.org/10.1021/acssensors.1c01991

\section{Document license:}

CC BY-NC-ND

DOI:

10.1021/acssensors.1c01991

Document status and date:

Published: 24/12/2021

\section{Document Version:}

Publisher's PDF, also known as Version of Record (includes final page, issue and volume numbers)

\section{Please check the document version of this publication:}

- A submitted manuscript is the version of the article upon submission and before peer-review. There can be important differences between the submitted version and the official published version of record. People interested in the research are advised to contact the author for the final version of the publication, or visit the $\mathrm{DOI}$ to the publisher's website.

- The final author version and the galley proof are versions of the publication after peer review.

- The final published version features the final layout of the paper including the volume, issue and page numbers.

Link to publication

\section{General rights}

Copyright and moral rights for the publications made accessible in the public portal are retained by the authors and/or other copyright owners and it is a condition of accessing publications that users recognise and abide by the legal requirements associated with these rights.

- Users may download and print one copy of any publication from the public portal for the purpose of private study or research.

- You may not further distribute the material or use it for any profit-making activity or commercial gain

- You may freely distribute the URL identifying the publication in the public portal.

If the publication is distributed under the terms of Article 25fa of the Dutch Copyright Act, indicated by the "Taverne" license above, please follow below link for the End User Agreement:

www.tue.nl/taverne

Take down policy

If you believe that this document breaches copyright please contact us at:

openaccess@tue.nl

providing details and we will investigate your claim. 


\title{
Sensing Methodology for the Rapid Monitoring of Biomolecules at Low Concentrations over Long Time Spans
}

\author{
Rafiq M. Lubken, Max H. Bergkamp, Arthur M. de Jong, and Menno W. J. Prins*
}

Cite This: ACS Sens. 2021, 6, 4471-4481

Read Online

ABSTRACT: Studies on the dynamics of biological systems and biotechnological processes require measurement techniques that can reveal time dependencies of concentrations of specific biomolecules, preferably with small time delays, short time intervals between subsequent measurements, and the possibility to record over long time spans. For low-concentration biomolecules, these requirements are very challenging since low-concentration assays are typically slow and require new reagents in every assay. Here, we present a sensing methodology that enables rapid monitoring of picomolar and subpicomolar concentrations in a reversible affinity-based assay, studied using simulations. We demonstrate that low-concentration biomolecules can be monitored with small time delays, short time

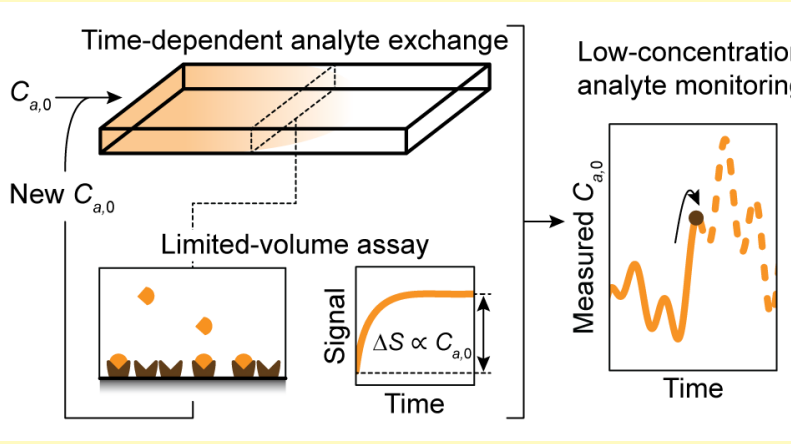
intervals, and in principle over an endless time span.

KEYWORDS: monitoring, sensing, low concentration, fast response, high precision, biomolecules, affinity kinetics

B iological systems and biotechnological processes exhibit time dependencies that are at the most basic level regulated by the dynamics of constituting biomolecules, such as small molecules, hormones, proteins, and nucleic acids. This calls for measurement technologies that allow the monitoring of biomolecular concentrations, for instance, to serve fundamental research on biological and biomedical dynamics, ${ }^{1-6}$ to enable the development of patient monitoring strategies based on real-time biomolecular data, ${ }^{7-10}$ and to enable the development of closed loop control strategies in biotechnological applications. ${ }^{11-16}$ Desirable characteristics of a generic monitoring technology are (1) precise and specific measurements, (2) small time delays between sampling input and data output, (3) short time intervals between successive measurements, and (4) a long total time span over which timedependent biomolecular concentration data can be recorded.

It is a fundamental challenge to develop a technology that can rapidly monitor low-concentration biomolecules over long time spans. Sensitive assays are available, such as ELISA and flow cytometry, ${ }^{17-20}$ but in these assays, new reagents are needed for every sample that is taken. The repeated consumption of reagents complicates applications where biomolecular concentrations need to be monitored over long time spans. On the other hand, sensing technologies that can operate without consuming reagents, such as surface plasmon resonance, $^{21}$ redox cycling, ${ }^{22}$ and quartz crystal microbalance, ${ }^{23}$ have not been designed for monitoring biomolecules at low concentrations, such as in the picomolar and subpicomolar range.
A generic principle to measure specific biomolecules at low concentrations is by using the biochemical affinity between specific binder molecules (such as antibodies and aptamers) and the biomolecule that is to be detected (the analyte). The specificity originates from molecular interactions such as charge, hydrogen bonding, van der Waals forces, and hydrophobic and steric effects. ${ }^{24}$ To be able to measure biomolecules at low concentrations with high precision, binder molecules are needed that have strong interactions with the analyte, which corresponds to high binding energies, low equilibrium dissociation constants $K_{\mathrm{d}}$, and low dissociation rate constants $k_{\text {off }}{ }^{25,26}$ However, this conflicts with the desire to have small time delays because low dissociation rate constants would imply a need for long incubation times to reach equilibrium. ${ }^{24-26}$ Furthermore, low dissociation rate constants result in a slow reversibility, which conflicts with the wish to enable short time intervals between successive measurements.

In this paper, a sensing methodology is presented that enables rapid monitoring of low-concentration biomolecules in the picomolar and sub-picomolar range, studied using simulations. The method is based on the use of binder molecules with a high affinity in a limited-volume assay, with a

Received: September 15, 2021

Accepted: November 15, 2021

Published: December 2, 2021 


\section{a Biomolecular monitoring}

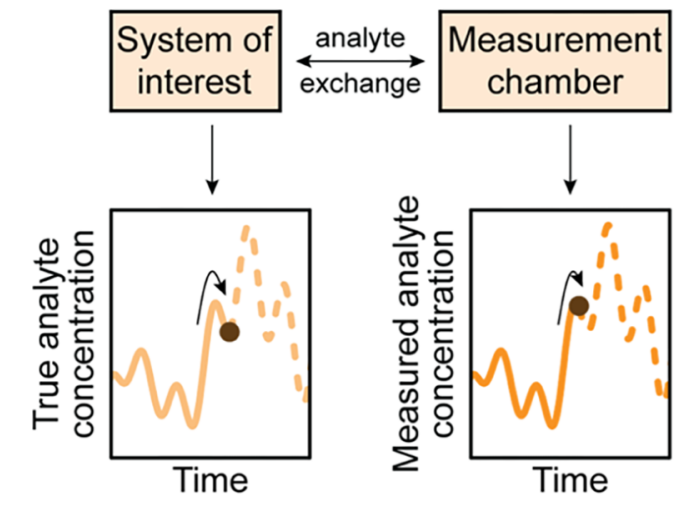

C

Infinite-volume assay

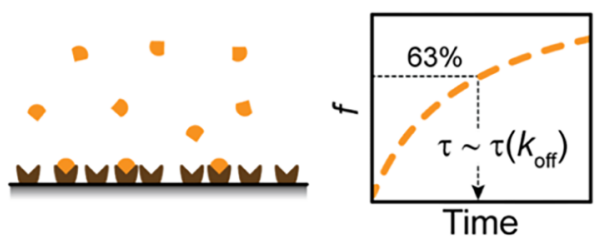

Limited-volume assay
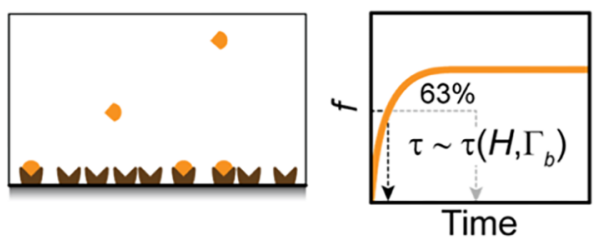

b

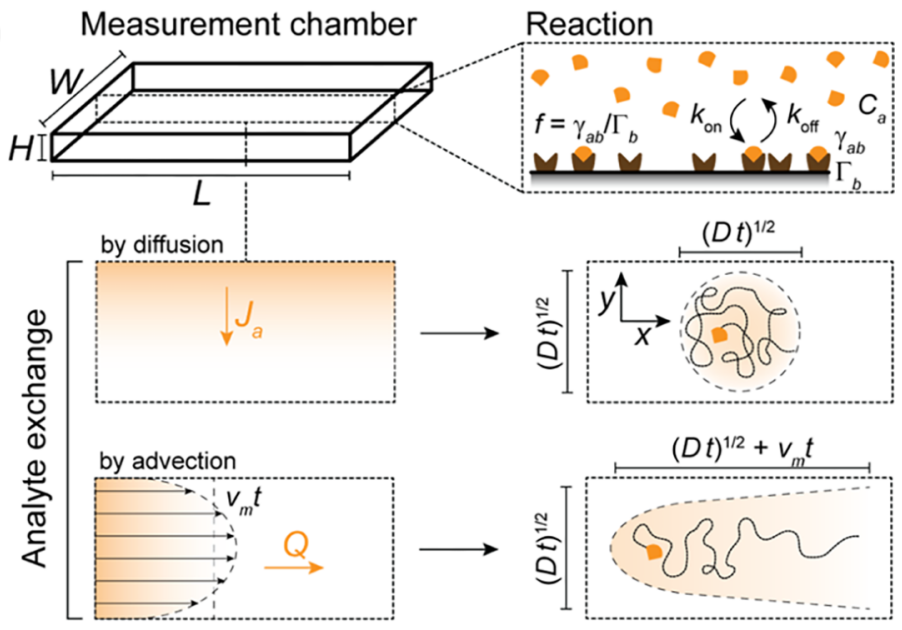

d

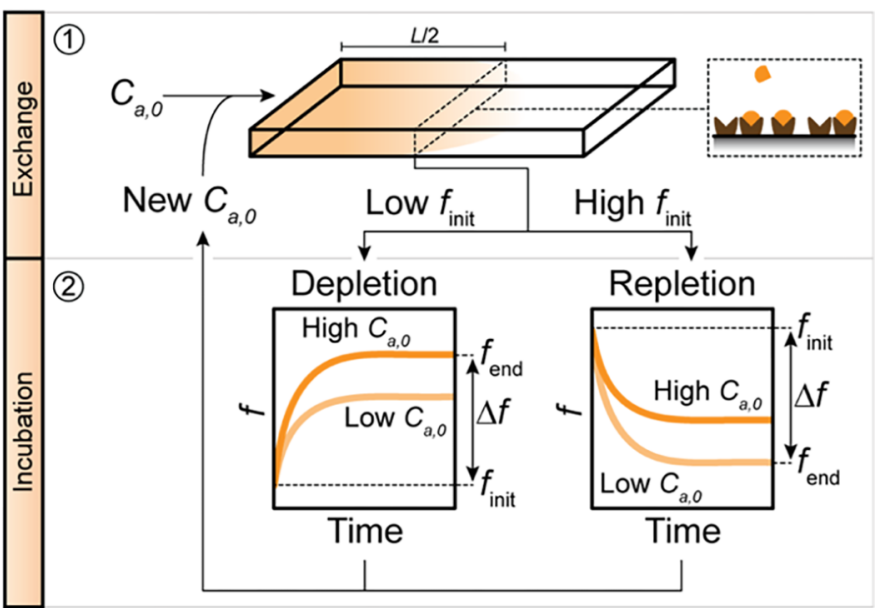

Figure 1. Concept of the sensing methodology for the rapid monitoring of low analyte concentrations. (a) Sensing system for biomolecular monitoring. Analyte molecules are exchanged between a biological or biotechnological system of interest and a measurement chamber. The data result in a concentration-time profile which should correspond as close as possible to the true analyte concentration-time profile in the system of interest. (b) Geometry of the measurement chamber, with height $H$, width $W$, and length $L$. A reaction rate at the sensor surface is caused by the association and dissociation between analyte molecules (orange) and binder molecules (brown), described by the association rate constant $k_{\text {on }}$, the dissociation rate constant $k_{\text {off }}$ the total binder density $\Gamma_{\mathrm{b}}$, the analyte concentration $C_{\mathrm{a}}$ near the sensor surface, and the density of analyte-binder complexes $\gamma_{\mathrm{ab}}$. Analyte exchange is facilitated by diffusion and advection, where diffusion occurs in both $x$ - and $y$-direction with the diffusion coefficient $D$, resulting in a net molar flux $J_{a}$, and where advection occurs in the $x$-direction only, with a developed flow profile with flow rate $Q$ and a mean flow speed $v_{\mathrm{m}}$. (c) Time profile of the sensor response for low analyte concentration $\left(C_{\mathrm{a}, 0}<K_{\mathrm{d}}\right)$, for two conditions: infinite-volume and limited-volume assays. For the infinite-volume condition, the time-to-equilibrium $\tau$ is determined by $k_{\text {off }}$, while for the limited-volume condition, $\tau$ is determined by the effective binder concentration. (d) Biomolecular monitoring using a limited-volume assay involves repeated cycles with two phases. In phase 1, analyte molecules are exchanged between the system of interest and the measurement chamber. In phase 2 , analyte molecules are not exchanged between the system of interest and the measurement chamber. The time-dependent signal is recorded in the middle of the measurement chamber at distance $L / 2$ from the entrance. The limited-volume condition gives a time dependence of the analyte concentration in the measurement chamber: a decrease over time (depletion) or an increase over time (repletion), depending on the input analyte concentration $C_{\mathrm{a}, 0}$ and the initial fractional occupancy $f_{\mathrm{init}}$ of binder molecules by analyte molecules. The input analyte concentration $C_{\mathrm{a}, 0}$ is derived from the measured time-dependent fractional occupancy $f$.

fully reversible detection principle and time-dependent sampling of the analyte of interest. The system allows optimal trade-offs between time characteristics and precision. We present the measurement concept, time dependencies of sensor signals, and a comprehensive analysis of the achievable time characteristics and precision as a function of sensor design parameters. We demonstrate that the sensing methodology enables precise quantification of low biomolecular concentrations, with time delays and interval times that are much shorter than the time dictated by the dissociation rate constant of the binder molecules. Furthermore, due to the reversible detection method, measurements can in principle be performed over an endless time span.

\section{BASIC CONCEPT OF THE SENSING METHODOLOGY}

The basic concepts of the sensing methodology are sketched in Figure 1. The sensing system features time-dependent sampling of the analyte of interest, provided by a timecontrolled analyte exchange between a biological or biotechnological system of interest and a measurement chamber (see Figure 1a). The measurement chamber contains specific 
Table 1. Standard Parameter Values Used in the Finite-Element Simulations ${ }^{a}$

\begin{tabular}{|c|c|c|c|}
\hline & parameter & value & description \\
\hline \multirow[t]{6}{*}{ input } & $H$ & $200 \mu \mathrm{m}$ & measurement chamber height \\
\hline & $D$ & $10^{-10} \mathrm{~m}^{2} \mathrm{~s}^{-1}$ & diffusion coefficient of the analyte molecule \\
\hline & $\Gamma_{\mathrm{b}}$ & $10^{-9} \mathrm{~mol} \mathrm{~m}^{-2}\left(600 \mu \mathrm{m}^{-2}\right)$ & binder density \\
\hline & $k_{\text {off }}$ & $10^{-4} \mathrm{~s}^{-1}$ & dissociation rate constant \\
\hline & $k_{\text {on }}$ & $10^{6} \mathrm{M}^{-1} \mathrm{~s}^{-1}$ & association rate constant \\
\hline & $C_{\mathrm{a}, 0}$ & $0.1 \mathrm{pM}$ & input analyte concentration \\
\hline \multirow[t]{6}{*}{ derived } & $\tau_{\mathrm{D}}=H^{2} / D$ & $400 \mathrm{~s}$ & characteristic diffusion time \\
\hline & $\tau_{\mathrm{R}, \mathrm{LV}}=\frac{1}{k_{\mathrm{on}} C_{\mathrm{b}, 0}}=\frac{H}{k_{\mathrm{on}} \Gamma_{\mathrm{b}}}$ & $200 \mathrm{~s}$ & characteristic reaction time for limited-volume assay with $C_{\mathrm{b}, 0} \gg C_{\mathrm{a}, 0}$ and $C_{\mathrm{b}, 0} \gg K_{\mathrm{d}}$ \\
\hline & $C_{\mathrm{b}, 0}=\Gamma_{\mathrm{b}} / H$ & $5 \mathrm{nM}$ & effective volumetric binder concentration \\
\hline & $K_{\mathrm{d}}=k_{\mathrm{off}} / k_{\mathrm{on}}$ & $100 \mathrm{pM}$ & equilibrium dissociation constant \\
\hline & $\alpha=\Gamma_{\mathrm{b}} / H K_{\mathrm{d}}=C_{\mathrm{b}, 0} / K_{\mathrm{d}}$ & 50 & $\begin{array}{l}\text { acceleration factor: reduction factor of the time-to-equilibrium of a limited-volume assay } \\
\text { with } \tau\left(H, \Gamma_{\mathrm{b}}\right) \text {, compared to an infinite-volume assay with } \tau\left(k_{\text {off }}\right)\end{array}$ \\
\hline & $D a=\tau_{\mathrm{D}} / \tau_{\mathrm{R}, \mathrm{LV}}=k_{\mathrm{on}} \Gamma_{\mathrm{b}} H / D$ & 2 & Damköhler number \\
\hline
\end{tabular}

${ }^{a}$ Details about the simulations are described in Note S4. Additional standard parameter values are given in Table S1 (see Note S1).

binder molecules from which signals are recorded. The data are translated into concentration-time profiles, which should resemble as close as possible the true concentration-time profile of analyte molecules in the system of interest. During the exchange of analyte molecules, various processes occur, such as mass transport by advection and diffusion and association and dissociation of analyte molecules to binder molecules (see Figure $1 \mathrm{~b}$ ). To illustrate the concept, a generic rectangular measurement chamber is assumed with height $H$, width $W$, and length $L$. The bottom surface is provided with binder molecules, where association and dissociation of analyte molecules occurs. The rates of association and dissociation depend on the association rate constant $k_{\text {on }}$, the dissociation rate constant $k_{\text {off }}$, the density $\Gamma_{\mathrm{b}}$ of binder molecules, and the analyte concentration $C_{\mathrm{a}}$ at the sensor surface. These processes result in a time-dependent density $\gamma_{\mathrm{ab}}$ of analyte-binder complexes, also represented as a fractional occupancy $f$ of binder molecules occupied by analyte molecules: $f=\gamma_{\mathrm{ab}} / \Gamma_{\mathrm{b}}$. Variables $\gamma_{\mathrm{ab}}$ and $f$ are changing as a function of analyte concentration and time. In an affinity-based sensor, the observed sensor signal scales with $f$; therefore, $f$ is used in this paper as the sensor readout parameter to determine the analyte concentration. Analyte exchange between the system of interest and the measurement chamber is facilitated by diffusion or a combination of diffusion and advection. A net diffusive molar flux $J_{\mathrm{a}}$ (orange gradient) is caused by concentration differences between the system of interest and the measurement chamber and by concentration differences within the measurement chamber. Advective mass transport of analyte molecules into the measurement chamber is facilitated by a developed laminar flow profile with flow rate $Q$ and mean flow speed $v_{\mathrm{m}}$ (black arrows). Here, it is assumed that diffusive transport occurs in both the longitudinal ( $x$-direction) and the lateral direction ( $y$-direction) and scales with the diffusion coefficient $D$, while advective transport occurs only in the longitudinal direction and scales with the mean flow speed $v_{\mathrm{m}}$.

Figure 1c sketches two different sensor designs, namely, an infinite-volume assay and a limited-volume assay. The graphs visualize the fractional occupancy $f$ of binder molecules occupied by analyte molecules as a function of time, with the corresponding characteristic time-to-equilibrium $\tau$, defined as the time needed to attain $63 \%$ of the difference between the starting level and the equilibrium level of $f$ (see Note S2). In an infinite-volume assay, continuous analyte exchange is enabled between the system of interest and the measurement chamber, where the system of interest is assumed to be much larger than the measurement chamber. The continuous analyte exchange could, for example, be facilitated by diffusive analyte transport across a contact area between the system of interest and the measurement chamber, while another configuration may involve a continuous flow of sample fluid provided into the measurement chamber from the system of interest. When the analyte exchange is effective and gives negligible time delay, then the analyte concentration at the sensor surface $\left(C_{\mathrm{a}}\right)$ is equal to the input analyte concentration $\left(C_{a, 0}\right)$. In the case of low analyte concentrations $\left(C_{\mathrm{a}, 0}<K_{\mathrm{d}}\right)$, the infinite-volume assay condition leads to a characteristic time-to-equilibrium $\tau$ $\cong 1 / k_{\text {off }}$ (see Note S2). This implies that the time-toequilibrium is determined by the dissociation rate constant $k_{\text {off }}$ so this time is long when the binder molecules strongly bind to the analyte molecules.

The sensor design with a limited-volume assay has very different properties. Here, analyte exchange between the system of interest and the measurement chamber is enabled for a limited time. After this analyte exchange, the binder molecules in the measurement chamber interact with only a limited sample volume and therefore with a limited amount of analyte molecules. Due to this limited volume, we can now define an effective volumetric concentration of binder molecules $C_{\mathrm{b}, 0}=\Gamma_{\mathrm{b}} / \mathrm{H}$, which is based on the number of binder molecules in the measurement chamber and the volume of the measurement chamber. When $C_{\mathrm{b}, 0}$ is high, with $C_{\mathrm{b}, 0}>$ $C_{\mathrm{a}, 0}$ and $C_{\mathrm{b}, 0}>K_{\mathrm{d}}$, then the time-to-equilibrium $\tau$ of the assay becomes dominated by the high concentration of binder molecules. When diffusional transport delays can be ignored, then the time-to-equilibrium of the assay equals $\tau \cong 1$ / $\left(k_{\mathrm{on}} C_{\mathrm{b}, 0}\right)$ (see Table 1 and Notes S1 and S2). Thus, the timeto-equilibrium of the limited-volume assay is determined by the association rate constant and the effective volumetric concentration $C_{\mathrm{b}, 0}$ of binder molecules, which leads to equilibrium timescales that are much shorter than the timeto-equilibrium of the infinite-volume assay.

In monitoring applications, it is preferred to record measurements with one and the same sensor over long time 
spans. To realize the limited-volume assay principle in a monitoring application, the sensor needs to be switched between two different conditions: an open condition and a closed condition. In the open condition, analyte molecules are exchanged effectively between the system of interest and the measurement chamber, as sketched in Figure 1a,b (also see Note S5). In the closed condition, analyte molecules are not exchanged between the system of interest and the measurement chamber, causing a limited-volume incubation in the measurement chamber, as sketched in the bottom graph of Figure 1c. We refer to the switching concept between the open and closed condition as "time-controlled analyte exchange". Figure $1 \mathrm{~d}$ illustrates the operating principle for a sensor where time-controlled analyte exchange is realized by a modulated flow. Phase 1 is the exchange phase, where the measurement chamber is supplied with the sample fluid so that the starting concentration in the chamber equals $C_{a, 0}$. Phase 2 is the incubation phase, where the exchange process is stopped so that the limited-volume assay condition is provided. During incubation under the limited-volume condition, the analyte concentration in the measurement chamber decreases over time (depletion) or increases over time (repletion), depending on the initial fractional occupancy $f_{\text {init }}$ of binder molecules by analyte molecules. When $f_{\text {init }}$ is low, the concentration of analyte molecules in the measurement chamber decreases over time, corresponding to the depletion of analytes. When $f_{\text {init }}$ is high, the concentration of analyte molecules in the chamber increases over time, corresponding to the repletion of analytes. For known $f_{\text {init, }}$ the supplied analyte concentration $C_{\mathrm{a}, 0}$ can be derived from the measured time-dependent fractional occupancy $f(t)$ during the incubation phase. At least two measurements need to be performed to determine the input analyte concentration $C_{\mathrm{a}, 0}$, for example, a measurement at the initial value $f_{\text {init }}$ and a measurement at the final value $f_{\text {end }}$ as indicated in the graph.

By sequentially applying cycles of open condition and closed condition, discrete samples with a limited volume are serially measured and result in time-dependent data that relate to the different samples supplied to the sensor. Each former measurement causes a varying nonzero initial fractional occupancy $f_{\text {init }}$ in the next measurement. The values of $f_{\text {init }}$ and $C_{a, 0}$ determine whether depletion or repletion occurs during the incubation phase. In the case of depletion, a higher input analyte concentration $C_{\mathrm{a}, 0}$ yields a larger, positive change in fractional occupancy $\Delta f=f_{\text {end }}-f_{\text {init }}$ since more analyte molecules are captured from solution. In the case of repletion, a higher $C_{a, 0}$ yields a smaller, negative change in fractional occupancy $\Delta f$ since less analyte molecules are repleted from the sensor surface into solution. An important property of the sensor is that the interactions between binder and analyte molecules are reversible. This gives the advantage that the limited-volume assay with time-controlled analyte exchange can be used over an endless time span.

In the next sections, we will study how sensor design parameters influence the time characteristics and precision of the sensing methodology. The time characteristics are quantified by finite-element simulations of mass transport in the sensor and reaction processes at the sensor surface, and the precision is quantified by calculating the stochastic variabilities in the measurements. The simulations and calculations are verified by experiments using a sensing technique with a singlemolecule resolution, called biosensing by particle mobility (BPM, see Note S7). ${ }^{27-29}$

\section{EXPERIMENTAL SECTION}

Finite-Element Analysis. Finite-element simulations were performed by solving diffusion, advection, and reaction equations simultaneously using COMSOL (COMSOL Multiphysics 5.5) and MATLAB (MATLAB R2019a, COMSOL Multiphysics LiveLink for MATLAB) (see Note S4). From the simulations, the time-toequilibrium $\tau$ was determined by calculating the time at which the analyte-binder complex density $\gamma_{\mathrm{ab}}$ is at $63 \%$ of the difference between the starting level and the equilibrium level of $\gamma_{\mathrm{ab}}$. The timecontrolled analyte exchange (see Figure 3) was simulated by instantaneously increasing/decreasing the analyte concentration throughout the measurement chamber $C_{\mathrm{a}}$ to $C_{\mathrm{a}, 0}$, with which a new measurement cycle starts. The density of analyte-binder complexes $\gamma_{a b}^{\text {start }}$ at the start of a cycle was set to be equal to the density of analyte-binder complexes $\gamma_{\mathrm{ab}}^{\text {end }}$ at the end of the preceding cycle. Sensor signals are reported at distance $L / 2$ in the measurement chamber (see Figure 1d). Precisions are reported at a distance $L / 2$ in the measurement chamber, where the signal is collected over a signal collection area of $1 \mathrm{~mm}^{2}$ (Figure $5 \mathrm{c}-\mathrm{e}$ ).

Fluid Cell Assembly. Glass slides $(25 \times 75 \mathrm{~mm}$, \#5, MenzelGläser) were cleaned by $40 \mathrm{~min}$ sonication in isopropanol (VWR, absolute) and twice by $10 \mathrm{~min}$ sonication in Milli-Q (Thermo Fisher Scientific, Pacific AFT 20). Subsequently, the glass slides were dried under nitrogen flow. A polymer mixture of PLL(20)-g[3.5]-PEG(2)

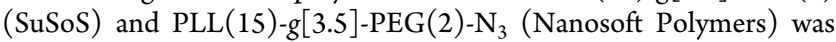
prepared at a final concentration of 0.45 and $0.05 \mathrm{mg} / \mathrm{mL}$ in Milli-Q, respectively. The glass slides were treated by oxygen plasma (Plasmatreat $\mathrm{GmbH}$ ) for $1 \mathrm{~min}$. A custom-made fluid cell sticker (Grace Biolabs), with an approximate volume of $20 \mu \mathrm{L}$, was attached to the glass slide and immediately filled with the polymer mixture. After $2 \mathrm{~h}$ of incubation, the polymer mixture was removed and the fluid cell was immediately filled with $0.5 \mathrm{nM}$ dsDNA tether solution (221 bp, with DBCO at one end and biotin at the other end) in $0.5 \mathrm{M}$ $\mathrm{NaCl}$ in phosphate-buffered saline (PBS). ${ }^{30}$ After overnight incubation, the solution in the fluid cell was exchanged by $2 \mu \mathrm{M}$ DBCO-functionalized dsDNA solution ${ }^{30}$ in $0.5 \mathrm{M} \mathrm{NaCl}$ in PBS and incubated for several days until use.

Particle Functionalization. A total of $2 \mu \mathrm{L}$ of streptavidinfunctionalized particles $(10 \mathrm{mg} / \mathrm{mL}$, Dynabeads MyOne Streptavidin $\mathrm{C} 1$, Thermo Fisher Scientific) was incubated with $1 \mu \mathrm{L}$ of biotinylated ssDNA binder molecules (10 $\mu \mathrm{M}$, IDT, HPLC purification) and $4 \mu \mathrm{L}$ of PBS for $70 \mathrm{~min}$. The particles were magnetically washed in $0.05 \mathrm{vol}$ \% Tween-20 (Sigma-Aldrich) in PBS and resuspended in $0.5 \mathrm{M} \mathrm{NaCl}$ in PBS to a final concentration of $0.1 \mathrm{mg} / \mathrm{mL}$ and sonicated using an ultrasonic probe (Hielscher).

BPM Assay. A total of $25 \mu \mathrm{L}$ of particle solution was added to the fluid cell and incubated for $10 \mathrm{~min}$. After incubation, the fluid cell was reversed causing unbound particles to sediment. After washing with $40 \mu \mathrm{L}$ of $0.5 \mathrm{M} \mathrm{NaCl}$ in PBS, $40 \mu \mathrm{L}$ of mPEG-biotin (500 $\mu \mathrm{M}$, PG1BN-1k, Nanocs) in $0.5 \mathrm{M} \mathrm{NaCl}$ in PBS was added to the fluid cell. After 15 min of incubation, the fluid cell was washed twice with $40 \mu \mathrm{L}$ of PBS. A mixture of ssDNA analyte molecules (IDT, standard desalting) and free binder molecules in PBS was added to the flow cell at the required concentration, immediately after preparation. The sample was observed under a white light source using a microscope (Leica DMI5000M) with a dark-field illumination setup at a total magnification of $10 \times$ (Leica objective, N plan EPI $10 \times / 0.25 \mathrm{BD}$ ). A field of view of approximately $1100 \times 700 \mu \mathrm{m}^{2}$ with a few thousand particles was imaged using a CMOS camera (FLIR, Grasshopper3, GS3-U3-23S6M-C) with an integration time of $5 \mathrm{~ms}$ and a sampling frequency of $30 \mathrm{~Hz}$. The particles were tracked by applying a phasorbased localization method. ${ }^{31}$ The particle activity was determined from the $x$ - and $y$-trajectories of all particles, by applying a maximumlikelihood multiple-windows change point detection algorithm. ${ }^{32}$ The particle activity at equilibrium and the time-to-equilibrium were extracted by fitting the measured particle activity over time using the equation given in Box S1. 

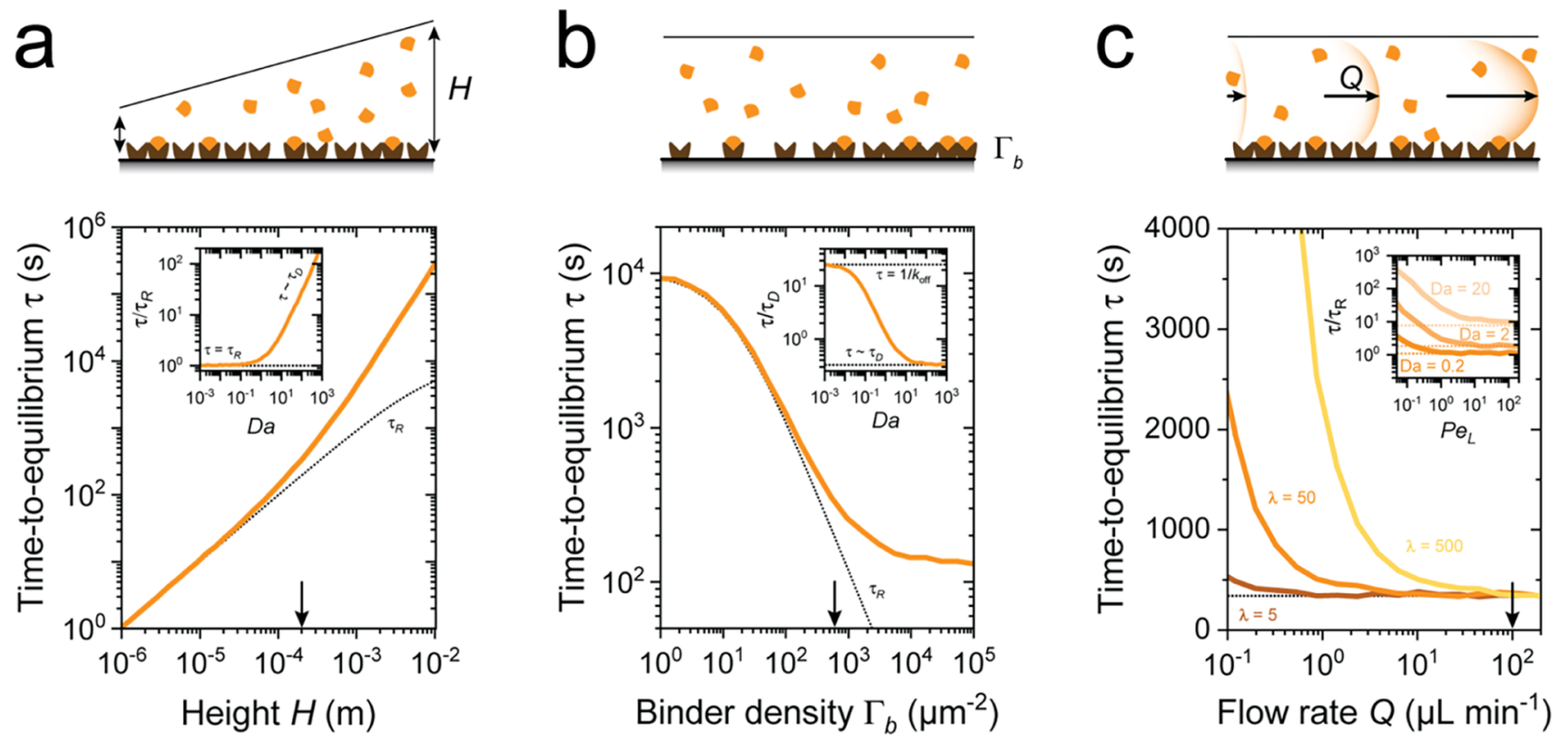

Figure 2. Time-to-equilibrium $\tau$ of a limited-volume assay for sensor designs with different heights, binder densities, and flow rates of analyte exchange. (a) Time-to-equilibrium $\tau$ as a function of measurement chamber height $H$ (orange line) for instantaneous analyte exchange. For small $H$, the observed $\tau$ is reaction-dominated (black dotted line), while for increasing $H$, the observed $\tau$ becomes diffusion-dominated. The inset shows the same data, where $\tau$ is normalized to $\tau_{\mathrm{R}}$ and plotted as a function of Damköhler number $D a$. The sketch above the graph visualizes a measurement chamber with an increasing $H$. (b) Time-to-equilibrium $\tau$ as a function of the binder density $\Gamma_{\mathrm{b}}$ (orange line) for an instantaneous analyte exchange. For low $\Gamma_{\mathrm{b}}$, the observed $\tau$ is reaction-dominated $\left(\tau=\tau_{\mathrm{R}}\right.$ black dotted line), while for increasing $\Gamma_{\mathrm{b}}$, the observed $\tau$ becomes diffusion-dominated. The inset shows the same data, where $\tau$ is normalized to the characteristic diffusion time $\tau_{\mathrm{D}}$ and plotted as a function of $D a$. The sketch above the graph visualizes a measurement chamber with an increasing $\Gamma_{\mathrm{b}}$. (c) Time-to-equilibrium $\tau$ as a function of flow rate $Q$ for three aspect ratios $\lambda=L / H$, for time-controlled analyte exchange by advection where the flow duration $t_{\text {exch }}$ equals the characteristic advection time $\tau_{\mathrm{A}}$. For small $Q$ the observed $\tau$ is limited by the advective transport of analyte molecules from the inlet toward the point of sensing at distance $L / 2$ from the inlet. For increasing $Q$ this transport process becomes faster causing the observed $\tau$ to be dominated by reaction and/or diffusion at high flow rates. The inset shows the same data $(D a=2)$ supplemented with $D a=0.2$ (reaction-limited) and $D a=20$ (diffusion-limited), where $\tau$ is normalized to $\tau_{\mathrm{R}}$ and plotted as a function of the longitudinal Péclet number $P e_{\mathrm{L}}$. The dotted lines show the $\tau / \tau_{\mathrm{R}}$ value at high $Q$ and are equal to the values found in panel a. The sketch above the graph visualizes a measurement chamber with an increasing $Q$. In all panels, the black arrows on the $x$-axis indicate the standard parameter values for $H, \Gamma_{\mathrm{b}}$, and $Q$ as listed in Table 1.

\section{RESULTS AND DISCUSSION}

Timescales of the Limited-Volume Assay. Figure 2 shows simulation results of the time-to-equilibrium of the limited-volume assay, for sensor designs with different measurement chamber heights, different binder densities, and different flow rates, assuming standard parameter values, as listed in Table 1. Figure 2a shows how the time-to-equilibrium $\tau$ depends on the measurement chamber height $H$, for a sensor with instantaneous analyte exchange (see Note S6 for the influence of analyte exchange on the sensor performance). The arrow on the $x$-axis indicates the height as listed in Table 1 . The data show that the time-to-equilibrium increases with the measurement chamber height. At small $H$, this increase is caused by a decrease in the effective volumetric binder concentration, while at large $H$, this increase is caused by diffusive transport limitations. The inset shows the same data, plotted as a function of the Damköhler number $\left(D a=\tau_{\mathrm{D}} / \tau_{\mathrm{R}, \mathrm{LV}}\right.$ $=k_{\mathrm{on}} \Gamma_{\mathrm{b}} H / D$, see Table 1$)$; low $D a$ means that the kinetics are limited by the reaction, and high $D a$ means that the kinetics are limited by diffusion. To achieve a fast time-to-equilibrium, the sensor should be designed with a large $C_{\mathrm{b}, 0}$, so a small $H$.

Figure $2 \mathrm{~b}$ shows how the time-to-equilibrium depends on the binder density $\Gamma_{\mathrm{b}}$, for a sensor with instantaneous analyte exchange. The arrow indicates the density, as listed in Table 1. For small $\Gamma_{\mathrm{b}}$, the time-to-equilibrium is long and determined by the dissociation rate constant $\left(\tau \cong 1 / k_{\text {off }}\right)$. For $\Gamma_{\mathrm{b}}>H K_{\mathrm{d}} \cong$
$20 \mu \mathrm{m}^{-2}$, the time-to-equilibrium decreases, until it stabilizes due to diffusive transport limitations $\left(\tau \cong \tau_{\mathrm{D}}=H^{2} / D\right)$. The inset shows the same data plotted as a function of $D a$. To achieve a fast time-to-equilibrium, the sensor should be designed with a large $C_{\mathrm{b}, 0}$, so a large $\Gamma_{\mathrm{b}}$.

Figure $2 \mathrm{c}$ shows how analyte exchange by advection contributes to the time-to-equilibrium per measurement cycle. The exchange phase involves a temporary flow of fluid into the measurement chamber, with flow rate $Q$ and duration $t_{\text {exch }}$ (see Note S6 for the influence of analyte exchange on the sensor performance). In the simulations, $t_{\text {exch }}$ was chosen to be equal to the characteristic advection time $\tau_{\mathrm{A}}=H L W / Q$ (see Table 1), which means that a total fluid volume equal to the volume of the measurement chamber is displaced. The time-toequilibrium $\tau$, which now includes a contribution $t_{\text {exch }}$ related to the exchange, is shown as a function of flow rate, for several values of the chamber aspect ratio $\lambda=L / H$. The arrow indicates the flow rate, as listed in Table 1 . For small $Q$ the observed $\tau$ is limited by $t_{\text {exch }}$, that is, the advective transport time of analyte molecules from the inlet toward the point of sensing at a distance $L / 2$ from the inlet, as sketched in Figure $1 d$. For increasing $\lambda$, that is, increasing $L$ with a fixed $H$, the time-to-equilibrium increases since $\tau_{\mathrm{A}}$ (and thus also $t_{\text {exch }}$ ) increases. For increasing $Q$ the time-to-equilibrium decreases, until it stabilizes at a level where the reaction and diffusion times determine the observed $\tau$. The inset shows the same data 

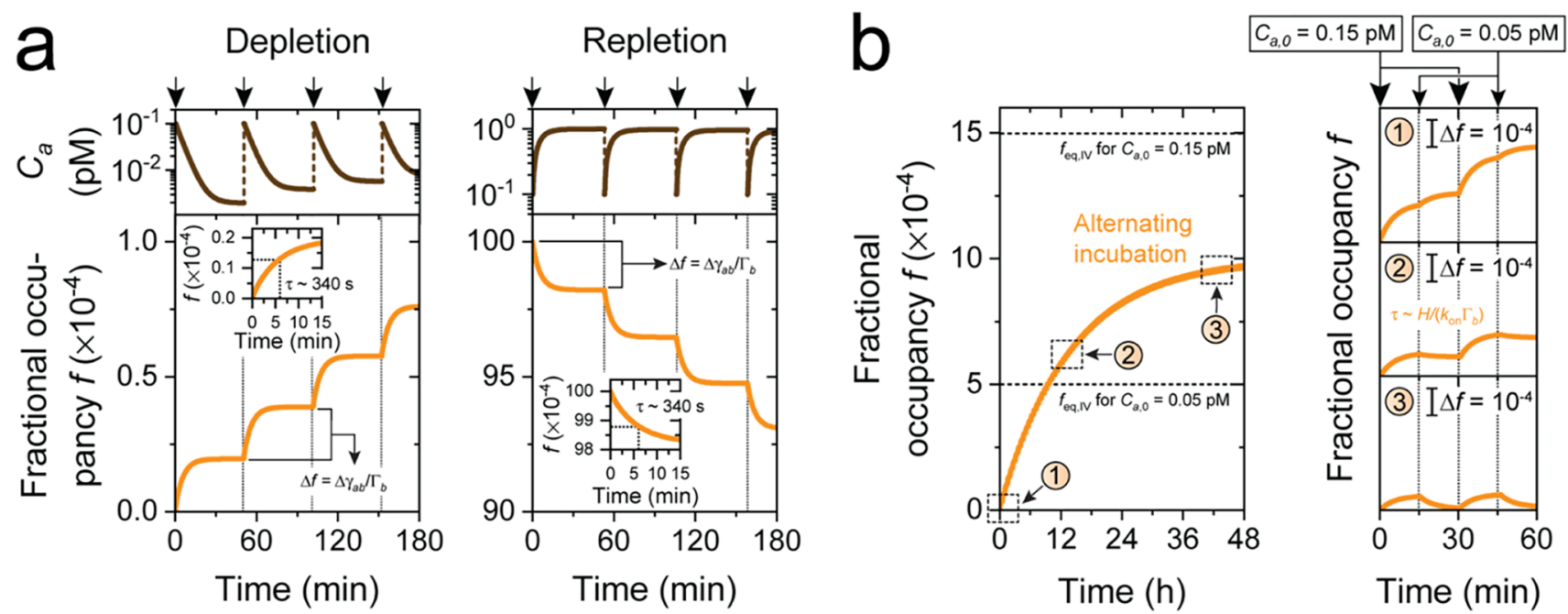

Figure 3. Simulated response of the biomolecular monitoring system using time-controlled analyte exchange. (a) Analyte concentration $C_{\mathrm{a}}$ in the measurement chamber (brown line) and the fractional occupancy $f$ of binder molecules by analyte molecules (orange line) as a function of time, for low $f_{\text {init }}$ and depletion of analyte in solution (left), and for high $f_{\text {init }}$ and repletion of analytes in solution (right). The dashed lines indicate time points where instantaneous analyte exchange occurs, where the bulk analyte concentration was set to $C_{\mathrm{a}, 0}=0.1 \mathrm{pM}$ after each period of approximately $50 \mathrm{~min}$. The insets highlight the kinetics of the first cycle, showing a time-to-equilibrium of $\tau=340 \mathrm{~s}$. For many cycles $(n \rightarrow \infty)$, both curves would approach $f_{\text {eq, IV }}=\frac{C_{a, 0}}{C_{a, 0}+K_{\mathrm{d}}}=10 \cdot 10^{-4}$, which equals the equilibrium value when an infinite volume is supplied (see Table S1). (b) Fractional occupancy $f$ as a function of time where cycles of analyte exchange and incubation are applied every 15 min with alternatingly $C_{\mathrm{a}, 0}=$ $0.15 \mathrm{pM}$ and $C_{\mathrm{a}, 0}=0.05 \mathrm{pM}$. The curve saturates at $f_{\mathrm{eq}, \mathrm{IV}}=10 \cdot 10^{-4}$, which equals the infinite-volume equilibrium value for the average concentration value $C_{\mathrm{a}, 0}=0.1 \mathrm{pM}$ (see Table $\mathrm{S} 1$ ). Dashed lines: continuous supply of $C_{\mathrm{a}, 0}=0.05 \mathrm{pM}$ yields $f_{\mathrm{eq}, \mathrm{IV}}=5 \cdot 10^{-4}$ and $C_{\mathrm{a}, 0}=0.15 \mathrm{pM}$ yields $f_{\text {eq,IV }}=15 \cdot 10^{-4}$ (see Table S1). The right panel shows zoom-in images of three sections of the solid curve, each representing four cycles of instantaneous analyte exchange and subsequent incubations of $15 \mathrm{~min}$. In zoom-in $1(t=0-1 \mathrm{~h})$, all curve segments show depletion behavior. In zoom-in images $2(t=12-13 \mathrm{~h})$ and $3(t=42-43 \mathrm{~h})$, depletion is seen for $C_{\mathrm{a}, 0}=0.15 \mathrm{pM}$, since $f_{\text {init }}<f_{\mathrm{eq}, \mathrm{IV}}\left(C_{\mathrm{a}, 0}=0.15 \mathrm{pM}\right)$, and repletion is seen for $C_{\mathrm{a}, 0}=0.05 \mathrm{pM}$, since $f_{\text {init }}>f_{\mathrm{eq}, \mathrm{IV}}\left(C_{\mathrm{a}, 0}=0.05 \mathrm{pM}\right)$. For all curve segments, the time-to-equilibrium $\tau=340 \mathrm{~s}$. The vertical scale bars indicate $\Delta f=$ $10^{-4}$.
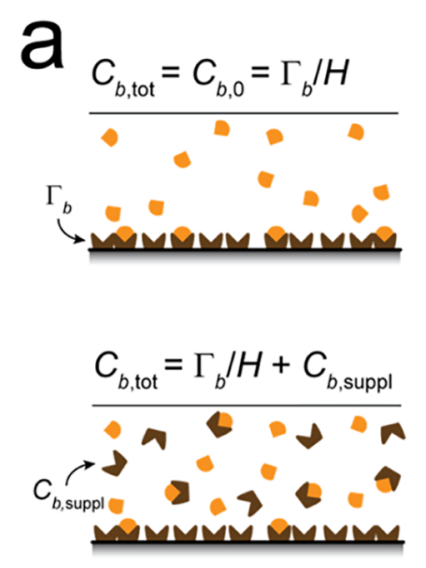
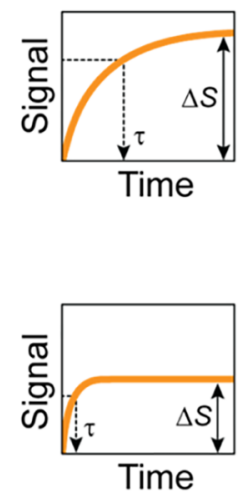

b

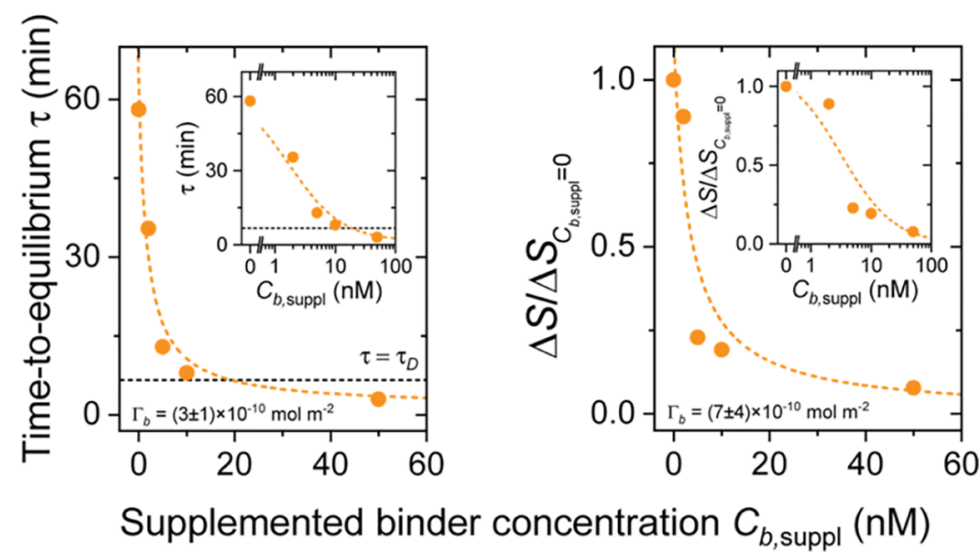

Figure 4. Experimental study of a limited-volume assay with varying binder concentrations using BPM. (a) Sketch of the measurement chamber in a BPM measurement (see Note S7) without (top) and with (bottom) supplemented binders with concentration $C_{\mathrm{b} \text {,suppl }}$. For simplicity, the particles of the BPM sensor are not shown in the sketch. (b) Experimentally observed time-to-equilibrium $\tau$ (left) and normalized signal change $\Delta S$ (right) as a function of supplemented binder concentration $C_{\mathrm{b} \text {,suppl }}$ in a BPM measurement. The model assay is based on DNA-DNA interactions, with ssDNA analytes at a concentration of $200 \mathrm{pM}$ (see Note S7). Left: the dashed line shows the fitted curve $\tau=p_{1} /\left(p_{2}+C_{\mathrm{b}, \text { suppl }}\right)+p_{3}$, where $p_{1}=1 /$ $k_{\text {on }}\left(k_{\text {on }}\right.$ is assumed to be equal for all binders), $p_{2}=\Gamma_{\mathrm{b}} / H$, and $p_{3}$ is the delay contributed by diffusion (see $\tau_{\mathrm{D}}$, black line, see also Figure $2 \mathrm{~b}$ ) and experimental steps (see the Experimental Section). Assuming $H=200 \mu \mathrm{m}$ (see Table 1 ), the fit gives $\Gamma_{\mathrm{b}}=(3 \pm 1) \cdot 10^{-10} \mathrm{~mol} \mathrm{~m}^{-2}$, which is comparable to the standard parameter value, as listed in Table 1 . The fitted association rate constant is $k_{\text {on }}=(1.5 \pm 0.4) \cdot 10^{5} \mathrm{M}^{-1} \mathrm{~s}^{-1}$, which is in the range of values reported in the literature for comparable DNA-DNA hybridization reactions. ${ }^{33,34}$ Right: In the depletion condition $\left(f_{\text {init }}<f_{\text {eq,IV }}\right)$, the fractional occupancy scales according to $f \propto 1 / C_{\mathrm{b}, \text { tot }}=C_{\mathrm{a}, 0} /\left(C_{\mathrm{b}, 0}+C_{\mathrm{b}, \text { suppl }}\right)$. The dashed line shows the fitted curve $\Delta S=p_{1} /\left(p_{2}+C_{\mathrm{b}, \text { suppl }}\right)$, where $p_{1}$ scales the change in fractional occupancy to signal change and $p_{2}=\Gamma_{\mathrm{b}} / H$. For $H=200 \mu \mathrm{m}$, it was found that $\Gamma_{\mathrm{b}}=(7 \pm 4) \cdot 10^{-10} \mathrm{~mol} \mathrm{~m}^{-2}$, which is comparable to the previously found value for $\Gamma_{b}$ and the standard parameter value, as listed in Table 1 . The insets show the same data on linearlogarithmic scales. The errors reported in the figure (smaller than the symbol size) and the caption are fitting errors based on a $68 \%$ confidence interval. 

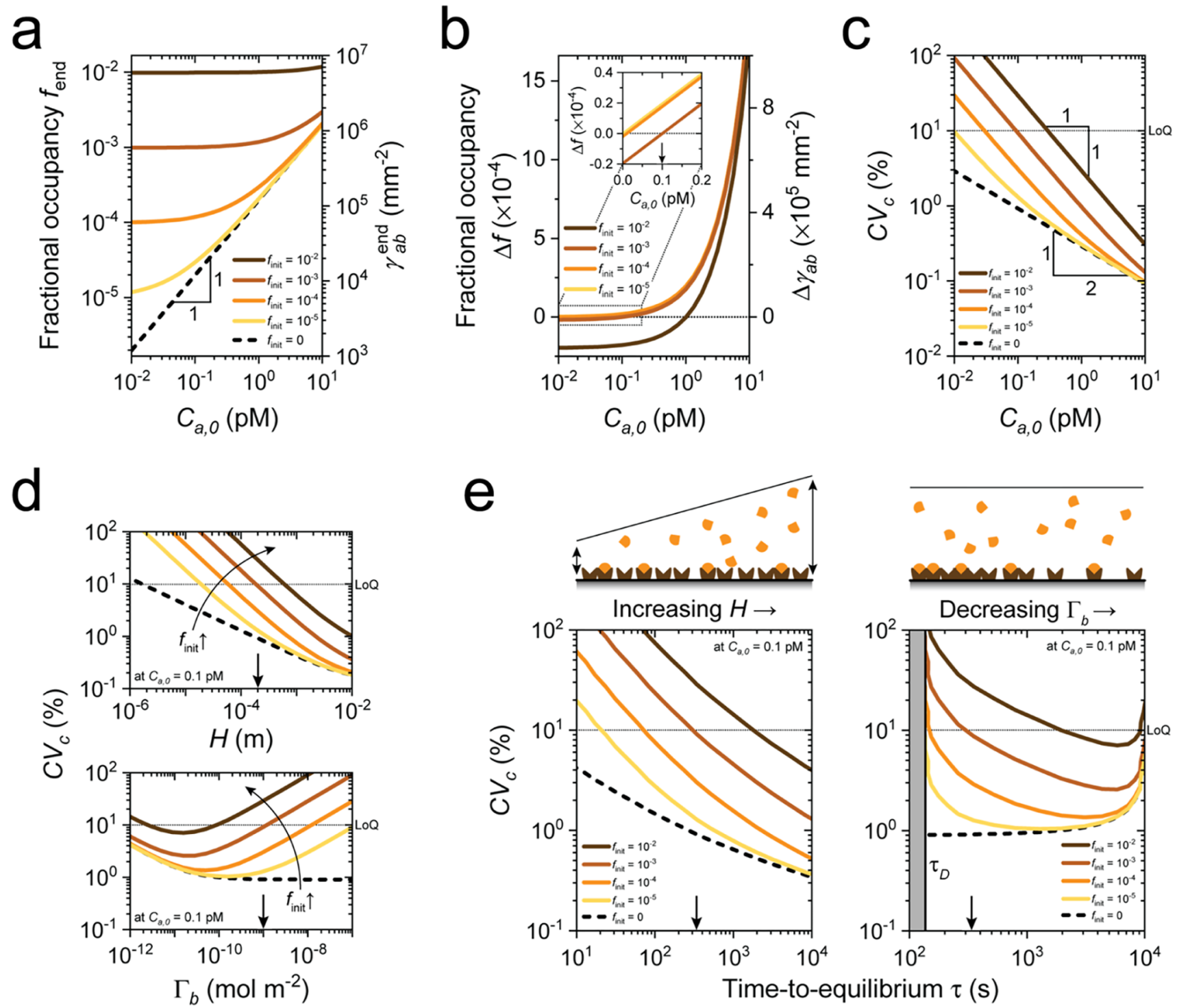

Figure 5. Analytical performance of the limited-volume assay, derived from simulations of a single measurement cycle. (a) Fractional occupancy at the end of the incubation $f_{\text {end }}$ as a function of analyte concentration $C_{\mathrm{a}, 0}$ for different initial fractional occupancies $f_{\text {init }}$. The right $y$-axis indicates the number of surface-bound analyte molecules at the end of the cycle $\gamma_{\mathrm{ab}}^{\text {end }}$. (b) Absolute change in fractional occupancy $\Delta f$ as a function of $C_{\mathrm{a}, 0}$ for various $f_{\text {init }}$. The right $y$-axis indicates $\Delta \gamma_{\mathrm{ab}}$. A positive $\Delta f$ and $\Delta \gamma_{\mathrm{ab}}$ indicate depletion; negative values indicate repletion. Note that the lines for $f_{\text {init }}$ $=10^{-3}$ and lower are overlapping. The inset shows the same data on a linear-linear scale. (c) Coefficient of variation $\mathrm{CV}_{\mathrm{C}}$ with which the analyte concentration $C_{\mathrm{a}, 0}$ can be determined as a function of analyte concentration $C_{\mathrm{a}, 0}$ for various initial fractional occupancies $f_{\text {init }} \cdot \mathrm{CV}_{\mathrm{C}} \mathrm{scales}$ as $1 / \sqrt{C_{\mathrm{a}, 0}}$ for low $f_{\text {init }}$ and high $C_{\mathrm{a}, 0} ; \mathrm{CV}_{\mathrm{C}}$ scales as $1 / C_{\mathrm{a}, 0}$ for high $f_{\text {init }}$ and low $C_{\mathrm{a}, 0}$. The horizontal dashed line indicates the $10 \%$ CV level that defines the LoQ (limit of quantification). (d) $\mathrm{CV}_{\mathrm{C}}$ as a function of measurement chamber height $H$ (top) and binder density $\Gamma_{\mathrm{b}}$ (bottom) for various initial fractional occupancies $f_{\text {init }}$ and $C_{\mathrm{a}, 0}=0.1 \mathrm{pM}$. The arrows on the $x$-axes indicate the standard parameter values for $H$ and $\Gamma_{\mathrm{b}}$, as listed in Table 1 . (e) $\mathrm{CV}_{\mathrm{C}}$ as a function of the observed time-to-equilibrium $\tau$ when varying the measurement chamber height $H$ (left) or binder density $\Gamma_{\mathrm{b}}$ (right) for various initial fractional occupancies $f_{\text {init }}$ and $C_{\mathrm{a}, 0}=0.1 \mathrm{pM}$. The sketches above the graphs visualize a measurement chamber with an increasing height or a decreasing binder density. The arrows on the $x$-axes indicate the obtained time-to-equilibrium using the standard parameter values for $H$ and $\Gamma_{\mathrm{b}}$, as listed in Table 1.

$(D a=2)$, supplemented with $D a=0.2$ (reaction-limited) and $\mathrm{Da}=20$ (diffusion-limited), plotted as a function of the longitudinal Péclet number $\left(P e_{\mathrm{L}}=\tau_{\mathrm{D}} / \tau_{\mathrm{A}}=Q / \lambda D W\right.$, see Table $\mathrm{S} 1)$; low $P e_{\mathrm{L}}$ means that the analyte exchange is limited by advection, and high $P e_{\mathrm{L}}$ means that the analyte exchange is limited by diffusion. A low $P e_{\mathrm{L}}$ causes a long time-toequilibrium due to slow mass transport by advection. Increasing $P e_{\mathrm{L}}$ results in a decrease in the time-to-equilibrium due to rapid filling of the chamber, until it stabilizes at a $\tau$ value equal to the value indicated in Figure 2a. Figure $2 \mathrm{c}$ shows the flow rate required to minimize the influence of the exchange process on the time-to-equilibrium. In the following sections, exchange with a high $P e_{\mathrm{L}}$ is assumed, that is, rapid filling of the measurement chamber without influence of the flow rate on the time-to-equilibrium.

Limited-Volume Assay with Time-Controlled Analyte Exchange. Figure 3 shows simulation results for a limitedvolume assay with time-controlled analyte exchange. The analyte exchange is assumed to be instantaneous and the incubation phase includes mass transport by diffusion and 
reaction kinetics within the measurement chamber itself but no analyte exchange between the system of interest and the measurement chamber. Figure $3 a$ shows data for repeated incubations with $C_{\mathrm{a}, 0}=0.1 \mathrm{pM}$. The analyte concentration $C_{\mathrm{a}}$ in the measurement chamber (brown line) and the fractional occupancy $f$ of the binders by analyte molecules (orange line) are plotted as a function of time, for conditions of analyte depletion (left) and analyte repletion (right). The time-toequilibrium $\tau$ of each incubation equals approximately $340 \mathrm{~s}$ (see Figure 2), having contributions from reaction $\left(\tau_{\mathrm{R}}=200 \mathrm{~s}\right.$ ) and diffusion $\left(\tau_{\mathrm{D}}=400 \mathrm{~s}\right)$. The contribution from the reaction to the time-to-equilibrium is much smaller than $1 / k_{\text {off }}=10^{4} \mathrm{~s}$, the value that would have been observed in the case of an infinite-volume assay (cf. Figure 1c). In the absence of diffusion limitations, the acceleration that can be achieved with a limited-volume assay compared to an infinite-volume assay equals $\alpha=\frac{1 / k_{\text {off }}}{\tau_{\mathrm{R}, \mathrm{LV}}}=\frac{k_{\mathrm{on}} \Gamma_{\mathrm{b}}}{H k_{\mathrm{off}}}=C_{\mathrm{b}, 0} / K_{\mathrm{d}}$, which clarifies how the speed of the assay is directly related to the ratio between effective volumetric binder concentration and the equilibrium dissociation constant.

Figure $3 \mathrm{~b}$ shows the response of a limited-volume assay with time-controlled analyte exchange for an analyte concentration that varies in time. As an example, the sensor is incubated with a time series of analyte concentrations that alternate around $0.1 \mathrm{pM}$ (orange line): the analyte concentration is alternatingly $C_{\mathrm{a}, 0}=0.05 \mathrm{pM}$ or $C_{\mathrm{a}, 0}=0.15 \mathrm{pM}$. The infinite-volume equilibrium fractional occupancy $f_{\text {eq, IV }}$ is given for $C_{\mathrm{a}, 0}=0.15$ $\mathrm{pM}$ and $C_{\mathrm{a}, 0}=0.05 \mathrm{pM}$ by the dashed black lines (see Table S1). The panels on the right show zoom-in images of the sensor response at three different time periods (starting at $t=$ 0,12 , and $42 \mathrm{~h}$ ). In all cases, the time-to-equilibrium is $\tau=340$ $\mathrm{s}=5.7 \mathrm{~min}$. Incubation with $C_{\mathrm{a}, 0}=0.15 \mathrm{pM}$ gives depletion behavior at all times (since $f_{\text {init }}<f_{\text {eq,IV }}$, top black line); for $C_{\mathrm{a}, 0}$ $=0.05 \mathrm{pM}$, depletion behavior is seen at $t<10 \mathrm{~h}$ and repletion at $t>10 \mathrm{~h}$ (when $f_{\text {init }}>f_{\text {eq,IV }}$, bottom black line).

Figure 4 shows an experimental study on how the time-toequilibrium in a limited-volume assay depends on the total binder concentration in the measurement chamber. Here, the total binder concentration has two contributions, namely, a contribution from surface-bound binders and a contribution from binders supplemented in solution. For detection, we made use of BPM, which is a biomolecular monitoring principle with single-molecule resolution. In the BPM sensor, the particles are transducers that record the binding of analyte molecules to specific binder molecules (see Note S7). Figure 4a shows a schematic representation of a measurement chamber with binder molecules present in the two forms: immobilized and nonimmobilized. Immobilized binder molecules are present with an effective volumetric concentration $C_{\mathrm{b}, 0}$. Binder molecules supplemented free in solution have concentration $C_{\mathrm{b}, \text { suppl }}$. In the absence of supplemented binder molecules (top), the total binder concentration in the measurement chamber equals $C_{\mathrm{b}, \text { tot }}=C_{\mathrm{b}, 0}=\Gamma_{\mathrm{b}} / \mathrm{H}$. In the presence of supplemented binder molecules (bottom), the total binder concentration equals $C_{\mathrm{b}, \text { tot }}=\Gamma_{\mathrm{b}} / H+C_{\mathrm{b} \text {,suppl }}$. Since the time-to-equilibrium of the reaction scales according to $\tau_{\mathrm{R}, \mathrm{LV}}$ $\propto 1 / C_{\mathrm{b}, \text { tot }}$ (see Table 1 ), an increasing supplemented binder concentration $C_{\mathrm{b} \text {,suppl }}$ results in a smaller $\tau$. Figure $4 \mathrm{~b}$ shows the measured time-to-equilibrium $\tau$ (left) and the signal change $\Delta S$ (right) as a function of $C_{\mathrm{b} \text {,supp }}$, for an analyte concentration of $200 \mathrm{pM}$ (see the Experimental Section). The data show that the time-to-equilibrium decreases for increasing $C_{\mathrm{b} \text {,suppl }}$. The measured signal change decreases with increasing supplemented binder concentration because only surface-captured analyte molecules generate a measurable signal. The dashed lines in Figure $4 \mathrm{~b}$ represent model fits (see the caption), demonstrating a good correspondence between model and experimental results. We conclude that the measurements of Figure 4 prove the basic concept of the sensing methodology proposed in this paper, namely, that a limited-volume design with time-controlled analyte exchange allows one to control the response time by tuning the concentration of binder molecules in the measurement chamber. Once the optimal binder concentration is known, the sensor can be made with binders immobilized in the measurement chamber. This will be a topic for future research.

Analytical Performance of the Limited-Volume Assay. Figure 5 shows how the analytical performance of the limited-volume assay depends on the sensor design. The results are based on numerical simulations with parameters, as listed in Table 1 . The analyte exchange is assumed to be instantaneous and the incubation includes mass transport by diffusion and reaction kinetics within the measurement chamber only. All panels show curves for different values of the initial fractional occupancy $f_{\text {init }}$ of the binder molecules.

Figure 5a shows the fractional occupancy of binders by analyte molecules at the end of the incubation $\left(f_{\text {end }}\right)$ as a function of the input analyte concentration $C_{\mathrm{a}, 0}$. For $f_{\text {init }}=0$ (dashed black line), $f_{\text {end }}$ scales linearly with the analyte concentration, which makes the sensor suitable for analyte quantification. For larger values of $f_{\text {init }}$ the curves start with a rather flat segment, from which one might erroneously conclude that under those conditions, low analyte concentrations cannot be determined. Interestingly, the limitedvolume assay has a linear dependence on concentration by focusing not on the absolute value of $f_{\text {end }}$ but rather on the change in fractional occupancy $\Delta f$ (see Note S2)

$$
\Delta f=f_{\text {end }}-f_{\text {init }} \cong \frac{H}{\Gamma_{\mathrm{b}}}\left(C_{\mathrm{a}, 0}-K_{\mathrm{d}} f_{\text {init }}\right)
$$

This equation shows that $\Delta f$ depends linearly on $C_{\mathrm{a}, 0}$, independent of the value of $f_{\text {init. }}$ This fact is also illustrated by the simulation results in Figure $5 b$. The response scales linearly with concentration $C_{a, 0}$ and is downshifted for increasing values of $f_{\text {init, }}$ in agreement with eq 1 (note that the steep increase in the curves relates to the logarithmic $x$-axis). Positive values of $\Delta f$ relate to depletion behavior and negative values to repletion. The curves cross the $x$-axis $(\Delta f=0)$ when $f_{\text {init }}$ corresponds to the equilibrium condition, that is, when there is no net association or dissociation during incubation because $f_{\text {init }}$ is equal to the equilibrium fractional occupancy of the infinite-volume case: $f_{\text {init }}=f_{\text {eq, IV }}=\frac{C_{a, 0}}{C_{a, 0}+K_{d}} \cong \frac{C_{a, 0}}{K_{d}}$. For example, the curve for $f_{\text {init }}=10^{-3}$ crosses $\Delta f=0$ at $C_{\mathrm{a}, 0}=f_{\text {init }} K_{\mathrm{d}}=$ $0.1 \mathrm{pM}$, as is highlighted in the inset of Figure $5 \mathrm{~b}$.

Figure $5 c$ shows the precision of the concentration output of the sensor, that is, the precision with which the analyte concentration in an unknown sample can be determined for a signal collection area of $1 \mathrm{~mm}^{2}$. The precision is calculated based on Poisson noise, which gives the fundamental limit of the precision that is achievable with a molecular biosensor due to stochastic fluctuations in the number of analyte molecules (see Note S3 and S8). ${ }^{35}$ To calculate the precision, a sensor with initial fractional occupancy $f_{\text {init }}$ is provided with a sample with analyte concentration $C_{\mathrm{a}, 0}$, resulting in a $\Delta f$ with 
variability $\sigma_{\Delta f}$, which via the slope of the calibration curve, given in Figure $5 b$, leads to a variability $\sigma_{\mathrm{C}}$ in the concentration output of the sensor (see Note S3). The precision is indicated as the concentration-based coefficient of variation $\mathrm{CV}_{\mathrm{C}}=\sigma_{\mathrm{C}}$ / $\mu_{\mathrm{C}}$, with $\sigma_{\mathrm{C}}$ being the variability and $\mu_{\mathrm{C}}$ being the mean of the concentration output. Figure $5 \mathrm{c}$ shows how the concentration precision depends on the analyte concentration and the initial fractional occupancy $f_{\text {init }}$. For $f_{\text {init }}=0$ (dashed line), the $\mathrm{CV}_{\mathrm{C}}$ scales as $1 / \sqrt{C_{a}, 0}$, in agreement with number fluctuations in a Poisson process (see Note S3). For higher $f_{\text {init }}$ a stronger dependency is observed $\left(\mathrm{CV}_{\mathrm{C}} \propto 1 / \mathrm{C}_{\mathrm{a}, 0}\right)$, caused by the smaller relative change in the fractional occupancy (see Note S3). The graph indicates the $10 \%$ precision level that is used to define the limit of quantification (LoQ) of the sensor (see Figure 5c, dashed horizontal line). The results show that analyte concentrations in the sub-picomolar range can be measured with a precision better than $10 \%$, even for high initial fractional occupancies.

Figure $5 \mathrm{~d}$ shows the precision of the concentration output of the sensor as a function of two design parameters, namely, the measurement chamber height $H$ (top panel) and the binder density $\Gamma_{\mathrm{b}}$ (bottom panel), at an analyte concentration $C_{\mathrm{a}, 0}=$ $0.1 \mathrm{pM}$, for an initial fractional occupancy $f_{\text {init }}$ between 0 and 0.01 . The arrows indicate the height and density, as listed in Table 1 . For an increasing $H$, a decrease in $C_{C}$ is observed, caused by an increase in the number of analyte molecules present in the measurement chamber. The $\mathrm{CV}_{\mathrm{C}}$ is the smallest for $f_{\text {init }}=0$ and increases for increasing $f_{\text {init }}$ since the absolute change in fractional occupancy decreases. The $\mathrm{CV}_{\mathrm{C}}$ decreases for increasing $\Gamma_{\mathrm{b}}$ caused by an increase in the number of analyte molecules captured from solution. The $\mathrm{CV}_{\mathrm{C}}$ reaches a plateau for $f_{\text {init }}=0$ due to a limited number of analyte molecules in the measurement chamber. For larger $f_{\text {init }}$ the absolute change in fractional occupancy decreases and causes a less precise concentration determination; this effect is in particular visible at high $\Gamma_{\mathrm{b}}$ where the absolute number of analyte-binder complexes increases due to $f_{\text {init }}$.

The trade-off between precision and time-to-equilibrium is illustrated in Figure 5e, for sensors with different heights of the measurement chamber (left) and different binder densities (right). The arrows indicate the time-to-equilibrium that results from the height and density, as listed in Table 1 . The left panel shows that an increase in $H$ gives, on the one hand, a slower sensor response (due to a larger diffusion distance) but, on the other hand, a lower $\mathrm{CV}_{\mathrm{C}}$ due to a larger number of analyte molecules present in the measurement chamber. At low $H$, the $\mathrm{CV}_{\mathrm{C}}$ strongly depends on $f_{\text {init }}$ due to the low number of analyte molecules in the solution. The right panel shows again that the $\mathrm{CV}_{\mathrm{C}}$ decreases for a slower sensor response, now controlled by decreasing the binder density $\Gamma_{\mathrm{b}}$. At high $\Gamma_{\mathrm{b}}$, the time-to-equilibrium is diffusion-limited (resulting in $\tau=130 \mathrm{~s}$ ). At low $\Gamma_{\mathrm{b}}$, the time-to-equilibrium is reaction-limited with $\tau=$ $1 / k_{\text {off }}=10^{4}$ (see Figure $2 \mathrm{~b}$ ). At high $\Gamma_{\mathrm{b}}$, the $\mathrm{CV}_{\mathrm{C}}$ increases for increasing $f_{\text {init }}$ due to the larger amount of analyte molecules on the sensor surface. At low $\Gamma_{b}$, the $\mathrm{CV}_{\mathrm{C}}$ strongly increases due to the small absolute change in the number of analyte-binder complexes during the incubation phase.

\section{CONCLUSIONS}

We have presented a sensing methodology suitable for monitoring low-concentration biomolecules with high precision, with small time delays and short time intervals, over an endless time span. The sensing methodology is based on a limited-volume assay, using high-affinity binders, a fully reversible detection principle, and time-controlled analyte exchange. We studied by simulations how the kinetics of the sensor depend on mass transport and on the surface reaction in the measurement chamber, and how time-controlled analyte exchange determines the system response and enables precise measurements of analyte concentration. Experimental results show the ability to control the sensor response time by tuning the total binder concentration in the measurement chamber. Finally, simulations show that the sensing principle allows picomolar and sub-picomolar concentrations to be monitored with a high precision over long time spans.

Approaches described in the literature for measuring lowconcentration biomolecules have focused primarily on assays in which every concentration determination involves consumption of reagents. ${ }^{17-20,36,37}$ When numbers of assays become high, due to frequent measurements over long time spans, then reagent-consuming approaches are complex and costly. The sensing methodology described in this paper is based on a fully reversible assay principle, without consuming reagents with each newly recorded concentration data point, enabling measurements with high frequency over an endless time span. The described assay principle can be implemented on several sensing platforms, for example, based on optical, electrical, or acoustical transduction methods, where especially sensing platforms with single-molecule resolution seem suitable since these allow digital measurements with very high precision, limited only by the number of observed molecular interactions. The sensing method is suited for the monitoring of a wide variety of analytes, including small molecules, proteins, and viral particles (see Note S9). Furthermore, the sensing methodology can be combined with various sampling methods, including remote advectionbased sampling through a sampling line or a catheter and proximal diffusion-based sampling methods for on-body and in-body monitoring devices. The presented sensing principle warrants further experimental studies, for example, to investigate trade-offs between time characteristics and precision, for various transduction methods, sampling methods, measurement chamber geometries, binder types, and for various complex biological matrices. Due to its sensing performance and generalizability, we believe that the limitedvolume assay with time-controlled analyte exchange will enable research on time dependencies of low-concentration biomolecules and novel applications in the fields of dynamic biological systems, patient monitoring, and biotechnological process control.

\section{ASSOCIATED CONTENT}

\section{Supporting Information}

The Supporting Information is available free of charge at https://pubs.acs.org/doi/10.1021/acssensors.1c01991.

Algebraic derivations of the used equations, finiteelement simulations, time-controlled analyte exchange, and BPM (PDF)

\section{AUTHOR INFORMATION}

\section{Corresponding Author}

Menno W. J. Prins - Department of Biomedical Engineering, Department of Applied Physics, and Institute for Complex Molecular Systems (ICMS), Eindhoven University of 
Technology, Eindhoven 5612 AZ, The Netherlands; Helia Biomonitoring, Eindhoven 5612 AZ, The Netherlands; ○ orcid.org/0000-0002-9788-7298; Email: m.w.j.prins@ tue.nl

\section{Authors}

Rafiq M. Lubken - Department of Biomedical Engineering and Institute for Complex Molecular Systems (ICMS), Eindhoven University of Technology, Eindhoven 5612 AZ, The Netherlands; $\odot$ orcid.org/0000-0001-7554-1141

Max H. Bergkamp - Department of Biomedical Engineering and Institute for Complex Molecular Systems (ICMS), Eindhoven University of Technology, Eindhoven 5612 AZ, The Netherlands; O orcid.org/0000-0001-8904-4322

Arthur M. de Jong - Department of Applied Physics and Institute for Complex Molecular Systems (ICMS), Eindhoven University of Technology, Eindhoven 5612 AZ, The Netherlands; (1) orcid.org/0000-0001-6019-7333

Complete contact information is available at: https://pubs.acs.org/10.1021/acssensors.1c01991

\section{Author Contributions}

R.M.L. and M.W.J.P. conceived and designed the methodology and measurement system. R.M.L. conceived, designed, and performed the simulations and the corresponding data analysis. M.H.B. performed the BPM experiments. R.M.L. and M.H.B. performed the BPM data analysis. R.M.L., A.M.d.J., and M.W.J.P. interpreted simulated data and discussed simulated results. All authors interpreted BPM experimental data and discussed BPM experimental results. R.M.L., A.M.d.J., and M.W.J.P. co-wrote the paper. All authors approved the submitted version of the manuscript.

\section{Notes}

The authors declare the following competing financial interest(s): M.W.J.P. and R.M.L. are listed as inventors on a filed patent application relating to the data presented in this paper. M.W.J.P. is cofounder of Helia Biomonitoring. All authors declare no further competing interests.

Data Availability: All data supporting the findings of this study are available from the corresponding author upon reasonable request.

Code Availability: All data analysis and simulation codes used for this study are available from the corresponding author upon reasonable request.

\section{ACKNOWLEDGMENTS}

We thank Yu-Ting Lin for her help with BPM experiments. Part of this work was funded by the Dutch Research Council (NWO), section Applied and Engineering Sciences, under grant number 16255. Part of this work was funded by the Safe$\mathrm{N}$-Medtech $\mathrm{H} 2020$ project under grant agreement number 814607.

\section{REFERENCES}

(1) Buszko, M.; Nita-Lazar, A.; Park, J.-H.; Schwartzberg, P. L.; Verthelyi, D.; Young, H. A.; Rosenberg, A. S. Lessons Learned: New Insights on the Role of Cytokines in COVID-19. Nat. Immunol. 2021, 22, 404-411.

(2) Fajgenbaum, D. C.; June, C. H. Cytokine Storm. N. Engl. J. Med. 2020, 383, 2255-2273.

(3) Duffy, D. Standardized Immunomonitoring: Separating the Signals from the Noise. Trends Biotechnol. 2018, 36, 1107-1115.
(4) Chen, P.; Huang, N.-T.; Chung, M.-T.; Cornell, T. T.; Kurabayashi, K. Label-Free Cytokine Micro- and Nano-Biosensing Towards Personalized Medicine of Systemic Inflammatory Disorders. Adv. Drug Delivery Rev. 2015, 95, 90-103.

(5) Young, A. T.; Rivera, K. R.; Erb, P. D.; Daniele, M. A. Monitoring of Microphysiological Systems: Integrating Sensors and Real-Time Data Analysis Toward Autonomous Decision-Making. ACS Sens. 2019, 4, 1454-1464.

(6) Zhang, Y. S.; Aleman, J.; Shin, S. R.; Kilic, T.; Kim, D.; Mousavi Shaegh, S. A.; Massa, S.; Riahi, R.; Chae, S.; Hu, N.; et al. Multisensor-Integrated Organs-on-Chips Platform for Automated and Continual In Situ Monitoring of Organoid Behaviors. Proc. Natl. Acad. Sci. U.S.A. 2017, 114, E2293-E2302.

(7) Heikenfeld, J.; Jajack, A.; Feldman, B.; Granger, S. W.; Gaitonde, S.; Begtrup, G.; Katchman, B. A. Accessing Analytes in Biofluids for Peripheral Biochemical Monitoring. Nat. Biotechnol. 2019, 37, 407419.

(8) Kim, J.; Campbell, A. S.; de Ávila, B. E.-F.; Wang, J. Wearable Biosensors for Healthcare Monitoring. Nat. Biotechnol. 2019, 37, 389-406.

(9) Heikenfeld, J.; Jajack, A.; Rogers, J.; Gutruf, P.; Tian, L.; Pan, T.; Li, R.; Khine, M.; Kim, J.; Wang, J.; et al. Wearable Sensors: Modalities, Challenges, and Prospects. Lab Chip 2018, 18, 217-248.

(10) Rodbard, D. Continuous Glucose Monitoring: A Review of Successes, Challenges, and Opportunities. Diabetes Technol. Ther. 2016, 18, S3-S13.

(11) Li, J.; Liang, J. Y.; Laken, S. J.; Langer, R.; Traverso, G. Clinical Opportunities for Continuous Biosensing and Closed-Loop Therapies. Trends Chem. 2020, 2, 319-340.

(12) Rawson, T. M.; O’Hare, D.; Herrero, P.; Sharma, S.; Moore, L. S. P.; de Barra, E.; Roberts, J. A.; Gordon, A. C.; Hope, W.; Georgiou, P.; et al. Delivering Precision Antimicrobial Therapy through ClosedLoop Control Systems. J. Antimicrob. Chemother. 2018, 73, 835-843.

(13) Mage, P. L.; Ferguson, B. S.; Maliniak, D.; Ploense, K. L.; Kippin, T. E.; Soh, H. T. A Closed-Loop Control of Circulating Drug Levels in Live Animals. Nat. Biomed. Eng. 2017, 1, 0070.

(14) Chemmalil, L.; Prabhakar, T.; Kuang, J.; West, J.; Tan, Z.; Ehamparanathan, V.; Song, Y.; Xu, J.; Ding, J.; Li, Z. Online/At-Line Measurement, Analysis and Control of Product Titer and Critical Product Quality Attributes (CQAs) During Process Development. Biotechnol. Bioeng. 2020, 117, 3757-3765.

(15) Wasalathanthri, D. P.; Rehmann, M. S.; Song, Y.; Gu, Y.; Mi, L.; Shao, C.; Chemmalil, L.; Lee, J.; Ghose, S.; Borys, M. C.; et al. Technology Outlook for Real-Time Quality Attribute and Process Parameter Monitoring in Biopharmaceutical Development - A Review. Biotechnol. Bioeng. 2020, 117, 3182-3198.

(16) Randek, J.; Mandenius, C.-F. On-Line Soft Sensing in Upstream Bioprocessing. Crit. Rev. Biotechnol. 2018, 38, 106-121.

(17) Todd, J.; Freese, B.; Lu, A.; Held, D.; Morey, J.; Livingston, R.; Goix, P. Ultrasensitive Flow-based Immunoassays Using SingleMolecule Counting. Clin. Chem. 2007, 53, 1990-1995.

(18) Rissin, D. M.; Kan, C. W.; Campbell, T. G.; Howes, S. C.; Fournier, D. R.; Song, L.; Piech, T.; Patel, P. P.; Chang, L.; Rivnak, A. J.; et al. Single-Molecule Enzyme-Linked Immunosorbent Assay Detects Serum Proteins at Subfemtomolar Concentrations. Nat. Biotechnol. 2010, 28, 595-599.

(19) Dunbar, S. A. Applications of Luminex xMAP Technology for Rapid, High-Throughput Multiplexed Nucleic Acid Detection. Clin. Chim. Acta 2006, 363, 71-82.

(20) Pinheiro, L. B.; Coleman, V. A.; Hindson, C. M.; Herrmann, J.; Hindson, B. J.; Bhat, S.; Emslie, K. R. Evaluation of a Droplet Digital Polymerase Chain Reaction Format for DNA Copy Number Quantification. Anal. Chem. 2012, 84, 1003-1011.

(21) Schasfoort, R. B. M.; Blum, S. R.; Eckman, J.; Abdiche, Y. N.; Karlsson, R.; Gedig, E. T.; Marquart, A.; Schuck, P.; Schasfoort, R.; Apiyo, D. Handbook of Surface Plasmon Resonance, 2nd ed.; Royal Society of Chemistry, 2017.

(22) Schoukroun-Barnes, L. R.; Macazo, F. C.; Gutierrez, B.; Lottermoser, J.; Liu, J.; White, R. J. Reagentless, Structure-Switching, 
Electrochemical Aptamer-Based Sensors. Annu. Rev. Anal. Chem. 2016, 9, 163-181.

(23) Durmuş, N. G.; Lin, R. L.; Kozberg, M.; Dermici, D.; Khademhosseini, A.; Demirci, U. Acoustic-Based Biosensors from Encyclopedia of Microfluidics and Nanofluidics; Springer, 2015.

(24) Atkins, P.; De Paula, J. Physical Chemistry for the Life Sciences; Oxford University Press, 2006.

(25) Kamat, V.; Rafique, A.; Huang, T.; Olsen, O.; Olson, W. The Impact of Different Human IgG Capture Molecules on the Kinetics Analysis of Antibody-Antigen Interaction. Anal. Biochem. 2020, 593, 113580 .

(26) Yang, D.; Singh, A.; Wu, H.; Kroe-Barrett, R. Dataset of the Binding Kinetic Rate Constants of Anti-PCSK9 Antibodies Obtained Using the Biacore T100, ProteOn XPR36, Octet RED384, and IBIS MX96 Biosensor Platforms. Data Brief 2016, 8, 1173-1183.

(27) Visser, E. W. A.; Yan, J.; van IJzendoorn, L. J.; Prins, M. W. J. Continuous Biomarker Monitoring by Particle Mobility Sensing with Single Molecule Resolution. Nat. Commun. 2018, 9, 2541.

(28) Yan, J.; van Smeden, L.; Merkx, M.; Zijlstra, P.; Prins, M. W. J. Continuous Small-Molecule Monitoring with a Digital Single Particle Switch. ACS Sens. 2020, 5, 1168-1176.

(29) Lubken, R. M.; de Jong, A. M.; Prins, M. W. J. Multiplexed Continuous Biosensing by Single-Molecule Encoded Nanoswitches. Nano Lett. 2020, 20, 2296-2302.

(30) Lin, Y.-T.; Vermaas, R.; Yan, J.; de Jong, A. M.; Prins, M. W. J. Click-Coupling to Electrostatically Grafted Polymers Greatly Improves the Stability of a Continuous Monitoring Sensor with Single-Molecule Resolution. ACS Sens. 2021, 6, 1980-1986.

(31) Martens, K. J. A.; Bader, A. N.; Baas, S.; Rieger, B.; Hohlbein, J. Phasor Based Single-Molecule Localization Microscopy in 3D (pSMLM-3D): An Algorithm for $\mathrm{MHz}$ Localization Rates using Standard CPUs. J. Chem. Phys. 2018, 148, 123311.

(32) Bergkamp, M. H.; IJzendoorn, L. J. v.; Prins, M. W. J. RealTime Detection of State Transitions in Stochastic Signals from Biological Systems. ACS Omega 2021, 6, 17726-17733.

(33) Bielec, K.; Sozanski, K.; Seynen, M.; Dziekan, Z.; ten Wolde, P. R.; Holyst, R. Kinetics and Equilibrium Constants of Oligonucleotides at Low Concentrations. Hybridization and Melting Study. Phys. Chem. Chem. Phys. 2019, 21, 10798-10807.

(34) Wetmur, J. G.; Davidson, N. Kinetics of Renaturation of DNA. J. Mol. Biol. 1968, 31, 349-370.

(35) Lubken, R. M.; de Jong, A. M.; Prins, M. W. J. How Reactivity Variability of Biofunctionalized Particles Is Determined by Superpositional Heterogeneities. ACS Nano 2021, 15, 1331-1341.

(36) Poudineh, M.; Maikawa, C. L.; Ma, E. Y.; Pan, J.; Mamerow, D.; Hang, Y.; Baker, S. W.; Beirami, A.; Yoshikawa, A.; Eisenstein, M.; et al. A Fluorescence Sandwich Immunoassay for the Real-Time Continuous Detection of Glucose and Insulin in Live Animals. Nat. Biomed. Eng. 2021, 5, 53-63.

(37) Wilson, B. D.; Soh, H. T. Re-Evaluating the Conventional Wisdom about Binding Assays. Trends Biochem. Sci. 2020, 45, 639649.

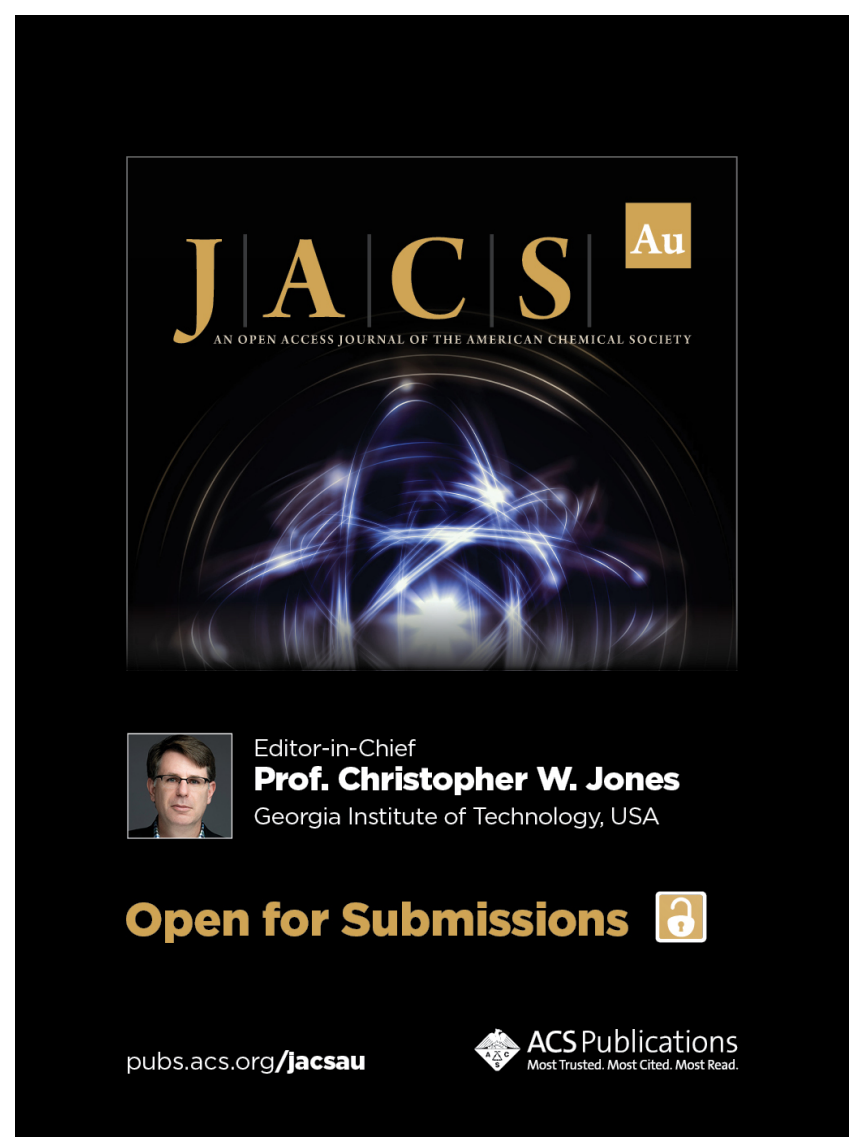

